labelled maximum dose and replacing with new inhalers too late for both reliever and controller medications. This is of clinical concern. The use of adhesive count stickers can help patients keep track of remaining doses objectively in a simple manner, with a good safety margin shown above. The use of the medication in the pMDIs can also be maximised with minimal wastage.

\section{IMPROVING COMPLETION RATES OF ELECTRONIC DISCHARGE SUMMARIES}

Anna Athanasia Ntovolou, Senel Hazal, Junaid Solebo. United Kingdom

\subsection{6/bmjpo-2021-RCPCH.238}

Background Annual Trust data and previous audits in our department revealed a massive number of incomplete electronic discharge summaries (EDSs) over the last three years. This posed a great risk to patient safety as important information about the patients' diagnosis, management and follow up arrangements are not shared with the GP, other healthcare professionals or the patients themselves when the EDS is not completed before discharge from hospital. Previous attempts to address this issue had been unsuccessful or temporarily effective.

Objectives We aimed to improve EDS completion rates in our Department and sustain this improvement through time, thus achieving enhanced patient care and safety.

Methods This is an ongoing project involving all inpatients discharged from the Paediatric ward of our District Hospital and all staff involved in their discharge process. Using the methodology of the Institute for Healthcare Improvement (IHI), multiple, small sample, Plan- Do- Study- Act (PDSA) cycles were performed monthly from September 2020. Changes were introduced gradually, and their effect tested with each cycle, as described below:

Cycle 1: Introduced ban on patients' departure from the ward without a hard copy of the EDS, via asking the nursing team to no longer facilitate this, nor expect the EDS to be mailed to them.

Cycle 2: Introduced the monthly EDS completion rates competition: each week's and weekend's ward leading Consultants were scored according to the EDS completion rates achieved during their week. Results were illustrated in a poster in the doctors' office, emailed to the Consultants and shared in the Junior Doctors' whatsapp group. The top performing leaders were commended on and rewarded with a chocolate prize.

Cycle 3: Increased study sample (days audited). Learning points about possible alterations in everyday work that could enhance EDS completion, shared with the team.

Cycle 4: Illustrated the month's results in a themed, eyecatching poster and circulated to the team comparison between the first and this month, demonstrating great improvement.

Cycle 5- currently ongoing: monthly audit of completed EDSs and information sharing with the team via means established during the previous cycles.

Results Significant improvement in the EDS completion rates (from $73 \%$ on average for each Consultant team on their worst audited day, to 95\%) was demonstrated throughout and has been so far sustained. Prolonged ward rounds and handovers, diminishing the time available to the team to complete
EDSs, were identified as possible factors for poor performance. Ambulating patients and mental health patients were most frequently the ones discharged without an EDS. Involving the Consultant body and turning the project into a game/ competition, while including both nurses and doctors, seemed to boost staff engagement in the process and minimise resistance to change.

Conclusions Early team involvement, at all levels of seniority, can enhance the success of quality improvement projects addressing longstanding day-to-day clinical problems. Continuous positive reinforcement and information sharing lead to better results and sustained improvement.

\section{ANCHOR PROGRAMME: PILOT HOME VISITATION PROGRAMME FOR CHILDREN WITH ADVERSE CHILDHOOD EXPERIENCES (ACES)}

Li Ming Ong, Padmini Yeleswarapu Sita, Jean Yin Oh, Helen Chen, Oh Moh Chay. Singapore

\subsection{6/bmjpo-2021-RCPCH.239}

Background Early childhood is a critical period for brain and biological development. Children with adverse childhood experiences (ACES) including maltreatment are at a high risk of developmental, behavioural and health problems lasting into adulthood. Anchor, a home visitation programme was commenced to support these children aged $0-3$ years old who have been maltreated and their families.

Objectives 1.To review the uptake of the families into the programme 2. To understand the profile of the children and their families enrolled into the programme

Methods Children enrolled into the programme undergo baseline evaluation of their health, development and behavior through ASQ-3 (Ages and Stages Questionnaire, Third Edition), ASQ: SE 2 (Ages and Stages Questionnaire: Social Emotional, Second Edition), CBCL (Child Behavior Checklist), YCPC (Young Child PTSD Checklist) and M-CHAT (Modified Checklist for Autism in Toddler, Revised). The primary caregiver undergoes evaluation of mental health through PHQ-9 (Patient Health Questionnaire-9) and GAD-7 (General Anxiety Disorder-7), and an assessment of bonding with the child using PICCOLO (Parenting Interactions with Children: Checklist of Observations Linked to Outcomes). After multidisciplinary team discussion of the above assessments, cases are tiered. The frequency of home visitation is determined based on the tiering of the case and the intervention plan is developed in discussion with the family using the principles of family centered practice. Their needs are identified early and supported with relevant home-based interventions. These children and their caregivers are also referred to medical and community services, as deemed necessary.

Results By December 2020, a total of 121 cases were referred to Anchor. 57 (42\%) of these cases are actively followed up by the programme. Approximately $15 \%$ of the referred families were not keen on the programme, while 3 of the families withdrew from Anchor. The remaining cases includes those that are pending enrolment and those rejected by the team as the family was being supported through other services. Physical abuse was the most common reason for referral. Approximately $50 \%$ of the children had a significant developmental delay (2 standard deviation) in at least 1 developmental 